\title{
Diagnostic Value of the Risk of Malignancy Index (RMI) for Detection of Pelvic Malignancies Compared with Pathology
}

Mojgan Karimi-Zarchi ${ }^{1}$, Shokouh Paymani Mojaver ${ }^{2}$, Mitra Rouhi ${ }^{3}$, Seyed Hossein Hekmatimoghaddam ${ }^{4}$, Reza Nafisi Moghaddam ${ }^{5}$, Pouria Yazdian-Anari ${ }^{6}$, Soraya Teimoori ${ }^{7}$

${ }^{1}$ Gynecology Oncology Fellowship, Associate Professor, Gynecology Department, Faculty of Medicine, Shahid Sadoughi University of Medical Sciences, Yazd, Iran

${ }^{2}$ General Practitioner, Shahid Sadoughi University of Medical Sciences, Yazd, Iran

${ }^{3}$ Medical Student, Young Researchers and Elites Club, Faculty of Medicine, Islamic Azad University, Yazd Branch, Yazd, Iran

${ }^{4}$ Pathologist, Assistant Professor, Department of Laboratory Sciences, Faculty of Paramedicine, Shahid Sadoughi University of Medical Sciences, Yazd, Iran

${ }^{5}$ Radiologist, Associate Professor, Department of Radiology, Faculty of Medicine, Shahid Sadoughi University of Medical Sciences, Yazd, Iran

${ }^{6}$ Medical Student, Student Research Committee, Faculty of Medicine, Shahid Sadoughi University of Medical Sciences, Yazd, Iran

${ }^{7}$ Medical Student, Young Researchers and Elites Club, Faculty of Medicine, Islamic Azad University, Yazd Branch, Yazd, Iran

\section{Type of article: Original}

\begin{abstract}
Introduction: Pelvic masses are among most the common causes of patient admission into gynecology clinics and one of the most common reasons for referral to gynecologic oncology departments due to the risk of uterine or ovarian malignancies. The aim of this study is to compare the four indices of the risk of malignancy index (RMI 1-4), as a combination of menstrual status, radiological findings, and serum CA125 concentration, for discrimination of benign from malignant pelvic masses.

Methods: This retrospective descriptive and analytic study was conducted on 200 patients with pelvic mass, post-surgery, and who were referred to the oncology department in Shahid Sadoughi hospital of Yazd (Iran) between June 2007 and September 2011. Data regarding demographics, pathology reports, paraclinical and clinical tests were analyzed. The four RMI indices were separately used for determination of benign vs. malignant masses using the optimized cutoff points, ROC curve, sensitivity, specificity, predictive value of positive and negative, and accuracy. Finally, p value for each index was calculated, and a final discrimination power was measured by using SPSS version 17 software.

Results: The calculated $\mathrm{p}$ values in the four RMI indices in ultrasound findings indicated statistical significance, and the RMI 2 showed the highest level of accuracy or diagnostic performance. RMI 2 had a cutoff point of 90, an under-chart area $86.7,79.36 \%$ sensitivity, $78.95 \%$ specificity, $58.44 \%$, positive predictive value, $90.08 \%$ negative predictive value, and $78.93 \%$ accuracy, and a $\mathrm{p}$ value of 0.004 . However, this relationship was found not to be meaningful using CT scan images.

Conclusions: Using RMI 2 for differentiation of malignant from benign pelvic masses is a reliable method with ultrasound findings.
\end{abstract}

Keywords: Pelvic mass, malignancy, CA125, risk, ultrasound, imaging

\section{Corresponding author:}

Pouria Yazdian-Anari, Student Research Committee, Faculty of Medicine, Shahid Sadoughi University of Medical Sciences, Yazd, Iran. Tel: +98.9385101025, Fax: +98.3536230354, E-mail: pouria_yazdiana_a@yahoo.com Received: July 06, 2015, Accepted: August 12, 2015, Published: November 2015 iThenticate screening: September 27, 2015, English editing: October 07, 2015, Quality control: November 01, 2015 (C) 2015 The Authors. This is an open access article under the terms of the Creative Commons Attribution-NonCommercialNoDerivs License, which permits use and distribution in any medium, provided the original work is properly cited, the use is non-commercial and no modifications or adaptations are made. 


\section{Introduction}

Pelvic masses are a common cause for admission of patients to gynecology clinics, and one of the most common reasons for referral to gynecologic oncology departments to assess the possibility of uterine or ovarian malignancies. A pelvic mass may present as an impalpable asymptomatic incidental finding or even a large mass extended from pelvis to abdominal cavity (1). The most prevalent type of pelvic masses is ovarian masses, which include cysts and tumors. The size of the mass, its mobility, consistency, shape, possible internal aqueous component, and associated pain are helpful features for diagnosis of the nature of the mass (2). A patient's first pelvic examination should be done at the age of 18 years or when sexual activity begins. Physical examination has sensitivity and specificity of $45 \%$ and $90 \%$, respectively (3). The increasing incidence of gynecologic malignancies necessitates the discovery of reliable ways to diagnose the benign/malignant nature of pelvic masses before surgery and confirmation by pathologic examination of biopsy specimens. Early diagnosis will result in earlier treatment, which result in a better prognosis and survival. Accurate assessment of mass status will also reduce unnecessary referrals for low-risk patients to gynecologic oncology departments. The importance of the risk of malignancy index (RMI), as an effective discriminating tool for this purpose has been widely accepted during the past decade. In 1995, Jacobs et al initially proposed the RMI as ultrasound findings $\times$ serum CA125 level $\times$ menstrual situation (menopause or not) (4). Tingulstad et al reported the extended index, as RMI 1 and RMI 2, in 1996 with further extension to RMI 1, RMI 2, and RMI 3 in 1999 (5). Goldstein described the approach, as well as available methods and techniques, for diagnosis of postmenopausal cystic adnexal masses (6). In 2009, Yamamoto et al suggested a new index as RMI 4, which adds consideration of tumor size as: "RMI $(1,2,3)=$ ultrasound findings $\times$ CA125 $\times$ menopausal status " $(7)$. For RMI 1, possible abnormal ultrasound findings (U) include multilocular cystic lesion, solid lesion, bilateralism, ascites, and metastasis. If nothing abnormal is found in the ultrasonographic report, $\mathrm{U}$ is regarded zero ( $\mathrm{U}=0$ ); if a single abnormality is seen, it will be $U=1$; and if 2 or more abnormal findings are seen, it will be $U=2$. Menopausal situation $(\mathrm{M})$ is either post-menopausal (more than one year since the last menstruation or age $>50$ if hysterectomy for any reason) $(M=3)$ or pre-menopausal $(M=1)(7-10)$. The serum concentration of CA125 is directly entered in the formula (4). In previous studies, a cutoff value of 200 for RMI 1 was suggested as the best discrimination point for segregation of benign and malignant pelvic masses, with high sensitivity and specificity levels (5). For RMI 2, U is 1 if there are $0-1$ abnormal findings and is 4 for 2 or more abnormal findings. $M$ would be 1 for pre-menopausal women and 4 in post-menopausal women. Again, the serum concentration of CA125 is directly entered in the formula (5). For RMI 3, U=1 for 0-1 abnormal findings and 3 for 2 or more abnormal findings. M=1 for premenopausal women and 3 for post-menopausal women. Again, the serum level of CA125 is directly entered in the formula (11). For RMI 4, the formula used is: $\mathrm{U} \times \mathrm{CA} 125 \times \mathrm{M} \times \mathrm{S}$, where $\mathrm{U}$ is 1 for $0-1$ abnormal findings and 4 for 2 or more abnormal findings. $M=1$ in pre-menopausal women and 4 for post-menopausal women. The serum level of CA125 is directly entered in the formula. S represents the largest diameter of the mass, which is 1 if $<7 \mathrm{~cm}$, and 2 if $\geqslant 7 \mathrm{~cm}$ (7). In this study we evaluated the ability of four malignancy risk indices (RMI 1, RMI 2, RMI 3, and RMI 4) to differentiate between benign or malignant pelvic masses based on both ultrasound and CT scan findings in patients referred to the gynecology referral center in Shahid Sadoughi hospital in Yazd, Iran.

\section{Material and Methods}

This retrospective, descriptive, and analytic study was implemented with women who were candidates for laparotomy due to pelvic masses and referred to Shahid Sadoughi Hospital, Yazd, Iran between June 2007 and September 2011. Before surgery, a detailed past history was gathered, all diagnostic modalities were completed, including complete clinical examination, ultrasonography, CT scan, and measurement of serum tumor markers, and the pathologic features of tumors were also recorded. The data was registered by questionnaire after taking signed written informed consent form from all participants and approval of the study by the institutional ethics committee. Using this information, four RMI values were determined using ultrasound findings, and also four independent RMI values were derived from CT scan findings for each patient. Data were analyzed using SPSS version 17 (SPSS Inc, Chicago, Illinois, USA). Findings were reported in ROC diagrams and the cutoff point determined. Predictive value of positive (PPV), predictive value of negative (NPV), sensitivity, specificity, area under the curve, and $p$ value were calculated using the histopathology report as the gold standard of diagnosis. Following this, the relationship between RMI and ultrasound and CT scans were compared to pathologic reports by the Mc-Nemar test.

\section{Results}

A total of 200 patients with ovarian tumors were enrolled in this study, of which, based on pathology reports, 143 patients $(71.5 \%)$ had benign tumors and 57 patients $(28.5 \%)$ had malignant lesions. The benign lesions were leiomyoma $(n=71)$, simple ovarian cyst $(n=24)$, endometrioma $(n=17)$, dermoid cyst $(n=14)$, serous cystadenoma 
$(\mathrm{n}=10)$, mucinous cystadenoma $(\mathrm{n}=3)$, adenomyosis $(\mathrm{n}=3)$, and cystadenofibroma $(\mathrm{n}=1)$, while the malignant tumors were serous cystadenocarcinoma $(n=26)$, mucinous adenocarcinoma $(n=14)$, germ cell tumor $(n=4)$, granulosa cell tumor $(n=4)$, uterine sarcoma $(n=3)$, clear cell tumor $(n=3)$, Krukenberg $(n=1)$, endometrioid tumor $(n=1)$, and cribriform tumor $(\mathrm{n}=1)$. The age distribution of patients is shown in Table 1. Table 1 shows that malignant pelvic tumors are associated with patient age. The highest rate occurring with 61-83 year old patients, while benign tumors are more common in 21-40 year old patients. From the 146 pre-menopausal patients involved in this study, 24 had lesions $(12 \%)$ that were malignant and $122(61 \%)$ had benign tumors, while with the 54 post-menopausal patients, $33(16.5 \%)$ had malignant lesions and $21(10.5 \%)$ had benign tumors. Therefore, it can be concluded that postmenopausal women are more likely to have cancer. Family history did not contain any cases of cancer for 185 patients, while in the remaining 15 cases a family history of ovarian cancer was found for six patients, breast cancer in four cases, lung cancer for three patients, and uterine malignancies in two cases. The first presentations of disease included pain (107 patients), abnormal uterine bleeding (AUB) (53 patients), gastrointestinal complaints (14 patients), palpable mass (13 patients), and incidental discovery of mass during ultrasonography (12 patients). The lowest level, mean, and maximum level of serum concentration of CA125 in benign neoplasms were $0.26,32.5$, and $275.9 \mathrm{U} / \mathrm{mL}$, respectively. In malignant tumors these figures were $3.41,139.79$, and $5000 \mathrm{U} / \mathrm{mL}$, respectively. Therefore, it can be concluded that CA125 is much higher in patients with malignancies.

Table 1. Distribution of patient age (divided into 4 groups)

\begin{tabular}{|l|l|l|l|}
\hline Age (years) & Benign Tumor, $\mathrm{n}(\%)$ & Malignant Tumor, $\mathrm{n}(\%)$ & Total, $\mathrm{n}(\%)$ \\
\hline$<20$ & $8(4)$ & $1(0.5)$ & $9(4.5)$ \\
\hline $21-40$ & $68(34)$ & $9(4.5)$ & $77(38.5)$ \\
\hline $41-60$ & $60(30)$ & $19(9.5)$ & $79(39.5)$ \\
\hline $61-83$ & $7(3.5)$ & $28(14)$ & $35(17.5)$ \\
\hline Total & $143(71.5)$ & $57(28.5)$ & $200(100)$ \\
\hline
\end{tabular}

The ultrasound features were documented for all 200 participants, but CT scans were done for only 100 patients. Ultrasonography findings showed that 143 (71.5\%) tumors were benign and $57(28.5 \%)$ tumors were malignant, as shown in Table 2. For determination of RMI, we used the area under the ROC curve to detect the cut off points of RMI 1, RMI 2, RMI 3, and RMI 4 with CT scans or ultrasonography. Table 3 shows the calculated values for different RMI indices based on ultrasound (upper row) and CT scans (lower row). Analysis shows that calculating RMI using ultrasonography can effectively differentiate benign from malignant tumors, and is less invasive compared with surgery and pathology. The highest diagnostic value occurs with RMI 2 (78.93), but analysis using CT scans is not effective using this methodology. There were significant differences between the mean of all 4 types of ultrasound-based RMI and surgery (Table 3), but the best results regarding diagnostic accuracy and area under the curve occur using RMI 2.

Table 2. Number of ultrasound findings in patients

\begin{tabular}{|l|l|l|l|}
\hline Number of Ultrasound Findings & Benign Tumor, $\mathrm{n}(\%)$ & Malignant Tumor, $\mathrm{n}(\%)$ & Total, $\mathrm{n}(\%)$ \\
\hline 0 & $4(2)$ & $7(3.5)$ & $11(5.5)$ \\
\hline 1 & $89(44.5)$ & $18(9)$ & $107(53.5)$ \\
\hline $2-5$ & $50(25)$ & $32(16)$ & $82(41)$ \\
\hline Total & $143(71.5)$ & $57(28.5)$ & $200(100)$ \\
\hline
\end{tabular}

Table 3. Values of parameters based on ultrasound (upper row) and CT scan (lower row) findings

\begin{tabular}{|l|l|l|l|l|l|l|l|l|}
\hline \multirow{2}{*}{ RMI } & $\begin{array}{l}\text { Cutoff } \\
\text { Point }\end{array}$ & $\begin{array}{l}\text { Area Under } \\
\text { the Curve }\end{array}$ & Sensitivity & Specificity & PPV & NPV & $\begin{array}{l}\text { Diagnostic } \\
\text { Accuracy }\end{array}$ & p Value \\
\hline \multirow{2}{*}{1} & 70 & 0.817 & 75.43 & 77.46 & 57.3 & 88.7 & 76.88 & 0.011 \\
\cline { 2 - 9 } & 74 & 0.804 & 71.79 & 74.13 & 65.11 & 79.62 & 73.19 & 0.557 \\
\hline \multirow{2}{*}{2} & 90 & 0.867 & 79.36 & 78.95 & 58.44 & 90.08 & 78.93 & 0.004 \\
\cline { 2 - 9 } & 100 & 0.851 & 77.79 & 81.03 & 68.29 & 80.35 & 75.25 & 0.536 \\
\hline \multirow{2}{*}{3} & 75 & 0.863 & 80.35 & 77.46 & 58.44 & 90.9 & 78.28 & 0.002 \\
\cline { 2 - 9 } & 80 & 0.843 & 73.68 & 77.58 & 68.29 & 81.81 & 76.4 & 0.672 \\
\hline \multirow{2}{*}{4} & 110 & 0.856 & 78.94 & 72.72 & 53.57 & 89.65 & 74.5 & 0.000 \\
\cline { 2 - 8 } & 200 & 0.822 & 66.66 & 82.75 & 72.22 & 87.68 & 76.28 & 0.876 \\
\hline
\end{tabular}




\section{Discussion}

Pelvic masses are one of the most common reasons for patients' referral to gynecologic oncology centers. The rate of malignancy in pelvic masses of pre-menopausal women is approximately $24 \%$ while in post-menopausal women it increases to more than $60 \%$, mostly from uterine or ovarian cancer (12). Unfortunately, most of these masses are asymptomatic or considered unimportant, leading to a delay in admission, difficulty of curative surgery, and ultimately decreased survival (2). In retrospective studies published during the past decade, the importance of estimating the risk of malignancy as an effective method for differentiation of malignant vs. benign masses was emphasized in an attempt to achieve early pre-operative diagnosis by using a combination of serum CA125 level, menstrual status (M), and ultrasound findings (U), the latter being composed of five characteristics (cystic multilocular lesion, solid lesion, bilateralism, ascites, and metastasis). Each of the above parameters has its own score defined for each of the four RMIs (11). Since the use of additional imaging, including CT scan or MRI, has not increased predictive accuracy compared to ultrasound examination, we decided to determine the specificity, sensitivity, predictive value of positive, predictive value of negative, and diagnostic accuracy of each RMI using ultrasound and CT scan, as compared with the result of pathologic examination. This study addresses the ability of the RMIs to discriminate between benign and malignant pelvic masses. The incidence of cancers in female reproductive organs is increasing, especially ovarian malignancies. Two-thirds of ovarian cancers are diagnosed only after metastasis or at stages 3 and 4, where the 5 -year survival drops from $95 \%$ in the initial stages to $28 \%$ in the late stages. Ovarian cancer is the 5 th most common cancer related cause of death for women, and has the highest mortality rate among female reproductive tract malignancies. Many researchers have tried to find an effective, reliable, easy, low-cost, and convenient method for pre-operative differentiation of benign from malignant masses, to help guide the patients with cancer toward subspecialty gynecologic oncology departments. The introduction of RMIs in recent studies aimed to apply this useful predictive tool in patients, provided that it has a meaningful statistical significance ( $\mathrm{p}$ value) (13).

Our data was processed using the SPSS software, versions 17, and ROC curves were obtained. Since the area under the curve in ROC determines the diagnostic power of test, its use for evaluation of a screening test for differentiation of benign from malignant masses is due to the curve coming from sensitivity vs. specificity and its basis on the range of variation of a parameter in successive cutoff points. The cutoff point is the shortest distance between peak of the curve and point 1 (14). Based on the cutoff points, a $2 \times 2$ table was made in which the following parameters are shown: sensitivity (the percent of patients with a pathologic finding of malignancy, which were initially assumed as having malignancy), specificity (the percent of patients with a pathologic finding of benign condition, which were initially assumed as having benign lesion), predictive value of positive (the percent of accurate positive diagnoses divided by the sum of true positive and false positive diagnoses), predictive value of negative (the percent of accurate negative diagnoses divided by the sum of true negative and false negative diagnoses), and diagnostic accuracy (the percent of accurately diagnosed patients divided by all of the patients). In a comparable study, Ulusoy et al. evaluated 296 patients with pelvic masses (mean age of 42 for benign masses and 47 for malignant ones), where 69\% $(n=205)$ had benign lesions (uterine leiomyoma being the most common) and $31 \%(n=91)$ had malignant tumors (the most common of them being ovarian mucinous cystadenocarcinoma). They found that the cutoff point for RMI was 153 and the index had the following characteristics: sensitivity $=76.4 \%$, specificity $=77.9 \%$, PPV $=65.9 \%$, and NPV=79.4\% (10). In a similar work, Yamamoto et al. studied 253 patients with pelvic masses (mean age for benign masses was 39 and 54 for malignant ones), where $84.2 \%(n=213$ ) proved to be benign and $15.8 \%(\mathrm{n}=40)$ proved to be malignant. All four RMI were determined, from which the RMI 4 had the highest (90.4\%) diagnostic accuracy with a cutoff point at 450. They also reported the following characteristics: sensitivity $=86.8 \%$, specificity $=91 \%$, PPV $=63.5 \%$, and NPV=97.5\%. For RMI 1, RMI 2, and RMI 3 the best cutoff point was found to be approximately 200, and the characteristics listed above were similar (7). During 2010, Van Den Akker et al. performed a study on 548 patients, with a mean age of 52 for those with benign lesion and 62 for those with malignant masses. This study involved 415 benign mass (76\%), 80 malignant mass (24\%), and 53 borderline malignancies (10\%). The most common benign and malignant masses were mucinous cysadenoma and serous cystadenocarcinoma, respectively. They calculated one RMI with a cutoff point of 200, at which the sensitivity, specificity, PPV, and NPV were $81 \%$, 85\%, 48\%, and 96\%, respectively (13). Additional research by Manjunath et al. was done on 152 patients with pelvic masses (mean age for benign masses was 45 and for malignant ones it was 49). Of these masses, 38.8\% ( $\mathrm{n}=62)$ proved to be benign (the most common was cysadenoma) and $61.2 \%(n=93)$ proved to be malignant (the most common being serous cysadenocarcinoma). Three RMI were checked without considerable difference in calculated parameters and in all RMIs the best cutoff point was at 200 (8). Obeidat et al. performed a study on 100 patients with pelvic masses. The mean age of those with 
benign masses ( $\mathrm{n}=28$, the most common of them being simple ovarian cyst) and malignant masses $(\mathrm{n}=72$, the most common was serous cysadenocarcinoma) were 50 and 58 years, respectively. The best cutoff point for RMI was determined to be 200 , in which the sensitivity, specificity, PPV, and NPV were $90 \%, 89 \%, 96 \%$, and $78 \%$, respectively (9). The diagnostic value of pelvic exams, ultrasounds and serum CA125 levels in women with pelvic masses was prospectively studied by Schutter et al., who found that the accuracy of these diagnostic tests in discriminating benign from malignant masses was nearly equal. In women with three negative parameters, no cancer was detected. Approximately $41.7 \%$ of the masses were malignant, while $55.7 \%$ were benign (3). Varras believed that ultrasound examination is not an independent factor for diagnosis of malignancy and imaging should not be regarded as the sole decision-making method for surgical intervention because radiological findings show considerable overlap between benign and malignant masses (15). A study by Strigini and coworkers showed that the diagnostic accuracy of ultrasound for diagnosis of malignancy in women with pelvic masses undergoing laparotomy is high, so that when both vaginal ultrasound exams and clinical exams are negative, malignancy can be ruled-out with a 70\% certainty (16). Another study by Van Trappen et al. in 2007 involved 196 cases of pelvic masses. Imaging techniques including ultrasound and MRI were assessed, and the study concluded that for RMI of 25-1000, the sensitivity and specificity of ultrasounds are $100 \%$ and $57 \%$ respectively, while the sensitivity and specificity of MRIs are $92 \%$ and $86 \%$, respectively (14). Yenen et al. stated in their 2012 study that RMI 4 with a cutoff point of 200 is the best RMI for anticipation of malignancy before surgery of borderline ovarian tumors (17). In the 2012 study by Anton et al., it was concluded that there is no difference between RMI, the risk ovarian malignancy algorithm, human epididymis protein 4 levels, and CA125 levels in discriminating between different types of ovarian tumors. RMI had the lowest sensitivity, but is the most commonly applied method. The HE4 showed the highest sensitivity for differentiation between ovarian malignancy and endometriosis. All of the parameters showed sensitivity and can be reliably used as a basis for patient referral (18). In this study, RMI 2 showed the best performance in predicting malignancy, compared with the other three indices. At the cutoff point 90 (above which the probability of malignancy of masses was high) RMI 2 had the most area under the curve (0.0865), showing the greatest concordance with pathologic results. However, when using CT scan findings, we found no meaningful correlation with any RMI. In Table 4, our results are compared with five other studies on the subject regarding the diagnostic parameters.

Table 4. Comparison of diagnostic parameters of this study with other similar works

\begin{tabular}{|l|l|l|l|l|l|l|}
\hline Parameter & Obeidat & Yamamoto & Van Den Akker & Manjunath & Ulusoy & Our study \\
\hline Sensitivity (\%) & 90 & 86.8 & 81 & 73 & 76.4 & 79.4 \\
\hline Specificity (\%) & 89 & 89 & 85 & 90 & 77.9 & 78.9 \\
\hline PPV (\%) & 96 & 97.5 & 48 & 93 & 65.9 & 58.4 \\
\hline NPV (\%) & 78 & 63.5 & 96 & 66 & 79.4 & 90.1 \\
\hline
\end{tabular}

\section{Conclusions}

Based on our study, RMI 2 use with ultrasound findings can be a useful and applicable method for initial assessment of patients with pelvic masses. Gynecologists may identify women with high probability of malignancy using the reasonably high sensitivity of RMI 2 and may separate patients with benign lesions who do not need immediate surgical intervention because of its high specificity. Thereby, unnecessary referrals to gynecologic oncologists will be prevented. In addition, oncologists may undertake the necessary decisions regarding type and technique of surgery by using RMI 2.

\section{Acknowledgments:}

The authors thank the staff in oncology department at Shahid Sadoughi hospital, Yazd, Iran for their assistance in data collection. No funding was taken for this study.

\section{Conflict of Interest:}

There is no conflict of interest to be declared.

Authors' contributions:

All authors contributed to this project and article equally. All authors read and approved the final manuscript.

\section{References}

1) Majmudar $T$, Abdel-Rahman H. Pelvic mass-diagnosis and management. Obstetrics, Gynaecology \& Reproductive Medicine. 2008;18(7):193-8, doi: 10.1016/j.ogrm.2008.05.004. 
2) Burbos N, Duncan TJ. Management of a pelvic mass. Obstetrics, Gynaecology \& Reproductive Medicine. 2010;20(11):335-40, doi: 10.1016/j.ogrm.2010.08.005.

3) Schutter EM, Kenemans P, Sohn C, Kristen P, Crombach G, Westermann R, et al. Diagnostic value of pelvic examination, ultrasound, and serum CA 125 in postmenopausal women with a pelvic mass. An international multicenter study. Cancer. 1994;74(4):1398-406, doi: 10.1002/10970142(19940815)74:4<1398::AID-CNCR2820740433>3.0.CO;2-J.

4) Jacobs I, Oram D, Fairbanks J, Turner J, Frost C, Grudzinskas J. A risk of malignancy index incorporating CA 125, ultrasound and menopausal status for the accurate preoperative diagnosis of ovarian cancer. BJOG: An International Journal of Obstetrics \& Gynaecology. 1990;97(10):922-9. doi: 10.1111/j.14710528.1990.tb02448.x

5) Tingulstad S, Hagen B, Skjeldestad FE, Onsrud M, Kiserud T, Halvorsen T, et al. Evaluation of a risk of malignancy index based on serum CA125, ultrasound findings and menopausal status in the pre - operative diagnosis of pelvic masses. BJOG: An International Journal of Obstetrics \& Gynaecology. 1996;103(8):826-31, doi: 10.1111/j.1471-0528.1996.tb09882.x.

6) Goldstein SR. Postmenopausal adnexal cysts: how clinical management has evolved. American journal of obstetrics and gynecology. 1996;175(6):1498-501, doi: 10.1016/j.ejogrb.2009.02.048.

7) Yamamoto Y, Yamada R, Oguri H, Maeda N, Fukaya T. Comparison of four malignancy risk indices in the preoperative evaluation of patients with pelvic masses. European Journal of Obstetrics \& Gynecology and Reproductive Biology. 2009;144(2):163-7, doi: 10.1016/j.ejogrb.2009.02.048. PMid: 19327881.

8) Manjunath A, Sujatha K, Vani R. Comparison of three risk of malignancy indices in evaluation of pelvic masses. Gynecologic oncology. 2001;81(2):225-9, doi: 10.1006/gyno.2001.6122. PMid: 11330953

9) Obeidat B, Amarin Z, Latimer J, Crawford R. Risk of malignancy index in the preoperative evaluation of pelvic masses. International Journal of Gynecology \& Obstetrics. 2004;85(3):255-8, doi: 10.1016/j.ijgo.2003.10.009. PMid: 15145261

10) Håkansson F, Høgdall EV, Nedergaard L, Lundvall L, Engelholm SA, Pedersen AT, et al. Risk of malignancy index used as a diagnostic tool in a tertiary centre for patients with a pelvic mass. Acta obstetricia et gynecologica Scandinavica. 2012;91(4):496-502, doi: 10.1111/j.1600-0412.2012.01359.x. PMid: 22229703

11) Morgante G, Marca A, Ditto A, Leo V. Comparison of two malignancy risk indices based on serum CA125, ultrasound score and menopausal status in the diagnosis of ovarian masses. BJOG: An International Journal of Obstetrics \& Gynaecology. 1999;106(6):524-7, doi: 10.1111/j.14710528.1999.tb08318.x.

12) Gillis C, Hole D, Still R, Davis J, Kaye S. Medical audit, cancer registration, and survival in ovarian cancer. The Lancet. 1991;337(8741):611-2, doi: 10.1016/0140-6736(91)91673-I

13) van den Akker PA, Aalders AL, Snijders MP, Kluivers KB, Samlal RA, Vollebergh JH, et al. Evaluation of the risk of malignancy index in daily clinical management of adnexal masses. Gynecologic oncology. 2010;116(3):384-8, doi: 10.1016/j.ygyno.2009.11.014. PMid: 19959215

14) Van Trappen P, Rufford B, Mills T, Sohaib S, Webb J, Sahdev A, et al. Differential diagnosis of adnexal masses: risk of malignancy index, ultrasonography, magnetic resonance imaging, and radioimmunoscintigraphy. International Journal of Gynecological Cancer. 2007;17(1):61-7, doi: 10.1111/j.1525-1438.2006.00753.x. PMid:17291233

15) Varras M. Benefits and limitations of ultrasonographic evaluation of uterine adnexal lesions in early detection of ovarian cancer. Clinical and experimental obstetrics \& gynecology. 2003;31(2):85-98.

16) Strigini FA, Gadducci A, Del Bravo B, Ferdeghini M, Genazzani AR. Differential diagnosis of adnexal masses with transvaginal sonography, color flow imaging, and serum CA 125 assay in pre-and postmenopausal women. Gynecologic oncology. 1996;61(1):68-72, doi: 10.1006/gyno.1996.0098. PMid: 8626120

17) Alanbay İ, Akturk E, Coksuer H, Ercan M, Karasahin E, Dede M, et al. Comparison of risk of malignancy index (RMI), CA125, CA 19-9, ultrasound score, and menopausal status in borderline ovarian tumor. Gynecological Endocrinology. 2012;28(6):478-82, doi: 10.3109/09513590.2011.633663. PMid: 22122561.

18) Anton C, Carvalho FM, Oliveira EI, Maciel GAR, Baracat EC, Carvalho JP. A comparison of CA125, HE4, risk ovarian malignancy algorithm (ROMA), and risk malignancy index (RMI) for the classification of ovarian masses. Clinics. 2012;67(5):437-41, doi: 10.6061/clinics/2012(05)06. 